\title{
A Case of Intractable Dysmenorrhoea
}

\author{
M F Shati ${ }^{1}$ N Begum², N Jahan ${ }^{3}$
}

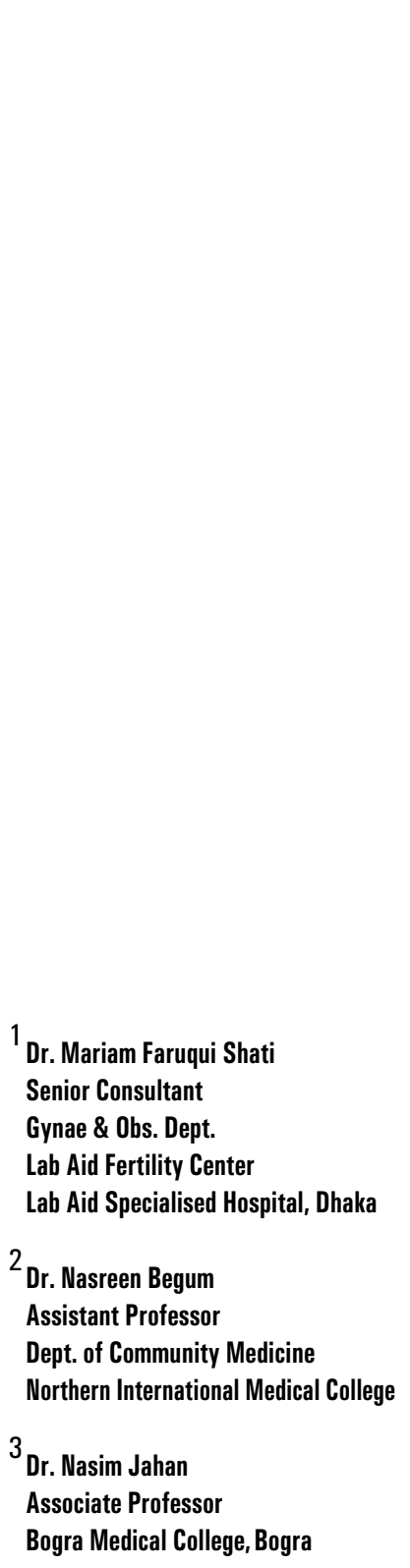

Correspondence

Dr. Nasreen Begum

Assistant Professor

Dept. of Community Medicine

Northern International Medical

College, Dhaka

\begin{abstract}
Dysmenorrhea (dysmenorrhoea or painful periods) is a medical condition of pain during menstruation that interferes with daily activities, defined by ACOG (American Congress of Obstetricians and Gynecologists). ${ }^{1}$ Still, dysmenorrhea is often defined simply as menstrual pain or at least menstrual pain that is excessive. ${ }^{2}$

A $19 y$ r old girl presented frequent dysmenorrhoea which was increasing in intensity and finally a lump was palpable on the left lower abdomen. She was treated surgically. A big mass size of a cricket ball was removed by laparotomy and patient was relieved from intractable dysmenorrhoea.
\end{abstract}

\section{Introduction :}

Dysmenorrhoea is quite common in early age and considered to be an under reported disorder. Incidence is $<50 \%$ in women population and $5-10 \%$ in adolescents. In about a fifth of these women, the pain is severe and can seriously disrupt normal activities. Pain is usually experienced in the 24 hours before menstruation or over the first 1 or 2 days of the period.

Dysmenorrhoea may be thought of as either primary or secondary. Primary dysmenorrhoea is where the symptoms occur in the absence of any demonstrable physical abnormalities, while secondary dysmenorrhoea occurs in association with some form of pelvic pathology. Only $<1 \%$ dysmenorrhoea occur due to defect in genital tract, it is rarely considered as a cause of dysmenorrhoea ${ }^{3}$.

Primary dysmenorrhoea occurs in young females with no pelvic pathology. It often begins with the onset of ovulatory cycles six months to one year after the menarche. ${ }^{1}$ The pain begins with the onset of the period and may last for 24-72 hours.

Secondary dysmenorrhoea occurs in association with some form of pelvic pathology. The pain typically precedes the start of the period by several days and may last throughout the period ${ }^{4}$. There may be associated dyspareunia. It may occur as a result of:

\section{o Fibroids \\ o Adenomyosis \\ 0 Endometriosis}

Secondary dysmenorrhoea is an indication of a separate disease which requires treatment other than simply pain relief using analgesics and that treatment of the underlying disorder will frequently reduce the problem of pain, or even remove the complaint altogether ${ }^{5}$

\section{Case Report}

A 19 year old domestic aid girl of poor socio-economic status was repeatedly suffering from dysmenorrhoea - which was gradually increasing in intensity. She had normal amount of flow. Her dysmenorrhoea used to occur on $2^{\text {nd }}$ and $3^{\text {rd }}$ day of her monthly period and gradually decreased in 3-4 days. It was so severe that she become unconscious due to pain and was admitted twice in a private clinic where she was treated medically.

On examination her nutritional status was found below average. She was mildly anemic and abdominal examination did not reveal any abnormalities.

There was no abnormality detected on Ultrasound (2D) therefore she was treated with mild analgesics but needed to switch on stronger NSAID. Still intensity of pain was increasing and she developed low grade fever with chronic lower abdominal pain. Hematological and Tuberculin tests were done but none of these tests were significant.

Re scan was also insignificant. She was thought a case of Endometriosis and put on oral contraceptive for 6 months.

Follow up during this period she was pain free, her general condition was improved. On with drawing oral contraceptive, her menstrual cycle was normal with mild dysmenorrhoea for three months. But from $4^{\text {th }}$ month onwards she again developed dysmenorrhoea. Lower abdominal examination a small firm lump was felt on left side. 4D scan revealed double uterus with collection in left uterine cavity. It was a big noncanalized horn of uterus. Ultrasonogram showed that vaginal cervical canal was in the same line with right uterine cavity.

Finally, concluded that it was a big noncommunicating rudimentary horn of uterus with hematometra. Endoscopic surgery (both laparoscopy and hysteroscopy) was not possible to carry out. Therefore laparotomy was done and per operative finding was a big mass of about a cricket ball attached with the left wall of right uterine mass. Tubes and ovaries were attached with right uterine body. The mass was dissected from its attachment. 
There was collection of blood in the cavity of left horn.

Right uterine cavity was not exposed during the operation. The patient had an uneventful post operative period, histopathology revealed uterine tissue. Since then her health was improved and she was free from intractable pain finally.

\section{Discussion}

Within a year or two of first menstrual period, 50 to 75 percent of young women begin to experience painful menstrual cramps, or dysmenorrhea, may diminish after the age of 25years or after childbirth. Dysmenorrhoea is very common although the precise incidence is not known, as it frequently goes unreported. Primary dysmenorrhoea is the most commonly given reason for absence from school amongst adolescent girls and approximately $15 \%$ will complain of severe dysmenorrhoea. Longer duration of menses, early menarche, smoking, alcohol and obesity are all risk factors associated with dysmenorrhoea. Females who are depressed and/or have poor social support networks are also more likely to experience pain ${ }^{6}$

Primary dysmenorrhea is more prevalent and is caused by prostaglandins and leukotrienes during ovulatory cycles. Endometrial prostaglandin levels increase during the luteal and menstrual phases of the cycle, causing uterine contractions. Treatment for primary dysmenorrhea includes pain relievers (analgesics) and drugs that block the action of prostaglandin (analgesics) These medications block the uterus from releasing prostaglandins, naturally occurring chemicals that cause cramps. ${ }^{8}$ Birth control pills (oral contraceptives) and other noncontraceptive hormones can relieve symptoms by suppressing ovulation.

Secondary dysmenorrhea is rare, and pain is associated with pelvic pathology (eg, bicornuate uterus, endometriosis, pelvic inflammatory disease, uterine fibroids). An underlying pelvic pathology (eg, endometriosis) or a uterine anomaly (eg, fibroids) may be present in about $10 \%$ of severe dysmenorrhea cases. ${ }^{5}$ If dysmenorrhoea starts after the age of 30 -endometriosis is a common cause. Uterine adenomyosis leiomyomas, polyps and an intra uterine device lead to secondary dysmenorrhoea. The abnormal muscle arrangement of bicornuate and septate uterus can give rise to intractable pain.

Dysmenorrhoea during adolescence caused by retention of menstrual flow are likely to occur in two types of anomalies, rudimentary uterine horn and unicornuate uterus with paramesonephric cyst. Rudimentary uterine horn develops due to failure of fusion of the mullerian ducts may result in two separate uterine bodies. Mal development of one body may create a small rudimentary horn. Some times this rudimentary horn may separate from the remainder uterus and does not communicate with the other uterine cavity or vagina.

When menstruation occurs blood cannot escape and is trapped in the rudimentary cavity resulting in severe dysmenorrhoea, haematometra or pyometra. If pregnancy occurs in a rudimentary horn, it may rupture which is fatal for both mother and fetus. Laparoscopy aided hysteroscopy is the single most useful diagnostic procedure. Treatment may be done at the same time. But initially noninvasive ultrasographic demonstration is useful, easily available and low cost procedure. MRI is undoubtedly the best for demonstrating congenital anomaly but hysterosulphingography may be a useful tool in some cases. ${ }^{7}$

Treatment of secondary dysmenorrhea depends on the underlying cause. A rudimentary horn is best excised especially before women conceive a child, if it is the site for haematometra. Reconstruction operation on bicornuate uterus usually gives unsatisfactory results. 5

In the present case patient had secondary dysmenorrhoea as she had a rudimentary horn -which gradually increased in size with the collection of blood with each menstrual cycle. Since it was non communicating. Haematometra along with the size of horn increased and caused intractable pain and subsequently a palpable mass developed in the pelvis.

Diagnostic imaging aid is final and early diagnosis. Ideally a rudimentary horn should be resected. The tube and ovary on the affected side can be preserved if blood supply is not impaired. 7

\section{References}

1. Patient Education Pamphlet : Dysmenorrhea By American Congress of Obstetricians and Gynecologists, retrieved January 2011(1)

2. TheFreeDictionary > dysmenorrhea Citing: Jonas: Mosby's Dictionary of Complementary and Alternative Medicine. Copyright 2005(4)

3. MedlinePlus > Painful menstrual periods Update Date: 9/2/2009 by: Susan Storck. Also reviewed by David Zieve(5)

4. Dysmenorrhea - Wikipedia, the free encyclopedia

en.wikipedia.org/wiki/Dysmenorrhe(2)

5. Proctor M, Farquhar C; Diagnosis and management of dysmenorrhoea. BMJ. 2006 May 13;332(7550):1134-8(3)

6. Alonso $\mathrm{C}$, Coe $\mathrm{CL}$; Disruptions of social relationships accentuate the association between emotional distress and menstrual pain in young women. Health Psychol. 2001 Nov;20(6):411-6

7. Jeffcoate's Principles of Gynaecology: International Edition with Colour Atlas revised and updated from 6th edition by Neerja Bhatla, Published in 2001, by Arnold; A member group of the hodder head line group; 338 Euston Road. London NW1 3BH; Dysmenorrhoea; Chapter 31 (585-587)

8. emedicine.medscape.com/article/953945-overview 